\title{
SIRI and the Origin of Epigenetic States in Saccharomyces cerevisiae
}

\author{
L. PILlus* AND J. RINE ${ }^{\dagger}$ \\ *Division of Biological Sciences, Section of Molecular Biology and UCSD Cancer Center, \\ University of California, San Diego, La Jolla, California 92093-0347; ${ }^{\dagger}$ Molecular and Cell Biology, \\ University of California, Berkeley, Berkeley, California 94720-3202
}

Silencing transcription of the majority of the genome in every cell is critical for normal growth, development, and regulation in multicellular eukaryotes. Current models of silencing build on the emerging details of chromatin modifications, as described in many of the papers in this volume. Modification states of histones, transcription factors, and other cellular proteins can strongly influence the recruitment and processivity of RNA polymerases.

Our goal in this short and decidedly noncomprehensive piece is to recap some insights from what has become a classic example of epigenetic control of transcription, that of silencing in the budding yeast Saccharomyces cerevisiae. Thorough and timely reviews may be found elsewhere (see, e.g., Rusche et al. 2003). Here, our historical and affectionate touchstone is the Silent Information Regulator gene, SIR1, which is pivotal in causing heterochromatin to assemble at and spread from silencers.

Most key players of silencing in yeast were initially identified mutationally. The subsequent molecular identification of some of their contributions to chromatin modification is mechanistically satisfying. Yet questions remain about other regulators, underscoring the probability of surprises in store as more is learned about how functionally different chromosomal states are established, maintained, and interconverted.

\section{MUTANTS OF SIR1 REVEAL EPIGENETIC SILENCING STATES}

Historically, a popular approach to transcriptional studies was the biochemical identification of molecules and machines required for activation. These analyses provided much detail as in vitro reconstitution of transcription on purified DNA templates became increasingly refined. However, contemporaneously, genetic analyses provided examples in which the absence of transcription, rather than its activation, is essential and most relevant for normal cell function. One such key example in yeast is the existence of silent copies of the mating-type information. The purpose of these copies is not to be expressed. Rather, these genes serve as sources of genetic information that, when transposed elsewhere, determine cell identity.

The elegant "cassette hypothesis" developed by Ira Herskowitz and his colleagues explained many aspects of the formal patterns through which cells could switch their identity by regulated access to the extra, although silent, copies of mating-type information (Herskowitz et al. 1977). Early versions of the hypothesis entertained the notion that something special about the mating-type locus, perhaps its promoter, caused expression at that locus. However, genetic studies soon turned the problem inside out with the discovery of recessive mutations that could activate the otherwise silent mating-type genes (Herskowitz et al. 1977; Haber and George 1979; Klar et al. 1979; Rine et al. 1979). What remained to be found, though, were the proteins necessary to prevent expression of the silent mating-type cassettes. Extended systematic analysis defined four major complementation groups of silent information regulators, the SIR genes, some alleles of which were also uncovered early on as STE, MAR, or CMT genes (Rine and Herskowitz 1987).

Even before molecular identification, the silent information regulator mutants fit into two categories: those with complete defects in silencing and one unusual case, sirl. In sir 1 mutants, silencing appeared to be only moderately affected in populations of mutant cells (Rine et al. 1979). Several different tests supported these distinctions between the mutants.

One way of assessing silencing is through mating tests in plate assays (Fig. 1). If haploid cells have normal silencing of the extra mating-type genes at $H M L$ and $H M R$, they mate with cells of the opposite mating type. If silencing is disrupted, haploids have many characteristics of diploid cells and fail to mate because they express mating-type information of both cell types. Under standard lab conditions, mating assays are performed so that the strains being tested have nonoverlapping auxotrophic markers. Therefore, mating is detected by selecting for prototrophic growth, which can occur only if a diploid has successfully formed by mating of the two haploids. In the example shown, sir $2 \Delta$ mutants are completely mating defective, whereas, sir $1 \Delta$ mutants appear to mate with an efficiency comparable to that of wild-type cells. However, this assay is blind to partial reductions in mating efficiency.

At first blush, a simple but incorrect explanation for the apparently incomplete effect on mating is that the sirl mutant alleles tested were only partial loss-of-function mutants, rather than null alleles that might completely destroy silencing. Therefore, when null alleles of SIR 1 were 


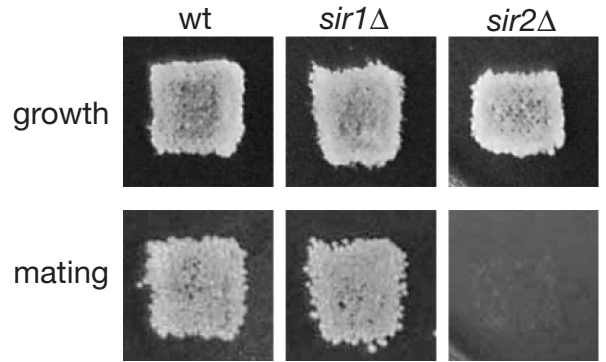

Figure 1. Silencing in populations of cells is easily assayed in patch mating tests. Wild-type and sir $\Delta$ mutant cells grow well, as shown in the control growth patches on rich medium in the top row. In mating tests for silencing, cells grow only if they successfully mate and form diploids with a wild-type cell of the mating type. In this example, as shown on the bottom row, sir $2 \Delta$ mutants are completely mating defective in comparison to wildtype and $\operatorname{sir} 1 \Delta$ cells. By other assays, sir 1 mutants were initially identified as silencing defective (Rine et al. 1979; Rine and Herskowitz 1987), although these defects are masked in population assays such as these. In quantitative mating assays or single-cell analyses (see text and Pillus and Rine 1989), 20-40\% of sirl $\Delta$ cells are silencing competent, whereas the remainder of the population appears as defective as $\operatorname{sir} 2 \Delta$ mutants.

found to have the same phenotype, all models based upon leaky alleles had to be discarded. Instead, two different views were equally compatible: (1) Sirlp played only a supporting role in silencing such that in all mutant cells the silent mating-type genes were activated to a slight extent or (2) in the absence of Sir1p, some cells silenced the $H M L$ and $H M R$ loci and some did not.

In 1989, after many happy and, at times, perplexing hours and days in the company of single sir $1 \Delta$ mutant cells at the micromanipulator, we concluded that SIR1 provided insight into important aspects of transcriptional regulation. Notably, epigenetic transcription states could be a fundamental part of the biology of single eukaryotic cells.

In normal haploid cells, with functional silencing, in the presence of mating pheromone of the opposite cell type, the cells undergo cell-cycle arrest and characteristic morphological alterations in preparation for mating. This acutely sensitive assay for transcriptional status allows silencing to be measured in single mutant cells, their siblings, and their progeny simply by examining them microscopically.

In "Epigenetic inheritance of transcriptional states in $S$. cerevisiae," we reported that seemingly identical cells from an isogenic population of sir $1 \Delta$ mutants had two decidedly distinct transcriptional states: those that appeared to be completely normal and silenced, and those that were as defective in silencing as their sir2, sir3, or sir 4 mutant brethren (Pillus and Rine 1989).

Importantly, cells of either sir $1 \Delta$ phenotypic class were mitotically stable with respect to their transcriptional silencing of mating-type information. This stability could last for many generations. Yet cells of either state, silenced or expressed, could also "switch" to assume the opposite phenotype at a low frequency, on the order of once in every 250 cell divisions. Therefore, it appeared that rather than existing as some sort of continuum or threshold phenomenon, silencing was an either/or, on/off biological switch.

Furthermore, either transcriptional state could be propagated through meiosis. There was not any requisite, wholesale reprogramming of silenced states to move successfully through this major developmental process. Because either on or off transcriptional state could be sustained in sirl mutants, we concluded that Sirlp was not necessary for the maintenance of silencing, but rather was more likely to play a key role in its establishment. This point has caused some confusion over the years. After all, the two populations observed in sirld cells could result from defects in either the establishment or the maintenance of the repressed state. In principle, one could imagine that establishment of silencing is ordinarily slow or inefficient in wild-type cells, but that maintenance is efficient, whereas maintenance is inefficient in sir $1 \Delta$ cells.

The link of SIR 1 function to establishment is supported by two additional observations. First, in the small subset of cells in which the silenced state is established in sir $1 \Delta$ cells, all cells in that generation are silenced rather than a small fraction. Second, under conditions in which SIR1 function can be restored, such as with regulated promoter fusions, silencing is efficiently restored to all the sir $1 \Delta$ cells in the population (Fox et al. 1997; Kirchmaier and Rine 2001).

\section{ADDITIONAL EPIGENETICALLY REGULATED LOCI REVEAL COMMON FEATURES}

As the molecular identification of SIR1 was being finalized (Stone et al. 1991), independent observations were made that the epigenetic transcriptional states revealed by $\operatorname{sir} 1 \Delta$ cells were not likely to be restricted to loss of SIR1. Indeed, regulatory site mutations at the silent mating-type loci, and expression of reporter genes at telomere-proximal sites revealed further fundamental possibilities for dynamic epigenetic changes in gene expression in yeast (Gottschling et al. 1990; Mahoney et al. 1991). The early studies of telomeric position effects paralleled those of sirl mutants and silent mating-type control. Indeed, both mitotic stability and distinct switches between states were observed in normal yeast cells. Telomeric silencing was also dependent on SIR2, SIR3, and SIR4, underscoring the potential coordinate function of these genes, although the epigenetic character of telomeric silencing is SIR 1 independent (Aparicio et al. 1991).

The possibility of an expanded role for SIR genes was also recognized in studies defining SIR2 as a suppressor of recombination between the repetitive array of rDNA repeats within the nucleolus (Gottlieb and Esposito 1989). Although yeast cells are ordinarily characterized by high levels of homologous recombination in both meiotic and mitotic cells, suppression of recombination in the rDNA helps assure optimal numbers of the repeats and thereby coordinates regulation of ribosomal inventories, protein synthesis, and growth. A recombinational silenc- 
ing role in the nucleolus is restricted to $\operatorname{SIR} 2$, since rDNA recombination is not affected in $\operatorname{sirl}, \operatorname{sir} 3$, or sir $4 \mathrm{mu}-$ tants. SIR2 suppresses not just recombination, but also transposition, and transcription of pol II-driven reporter genes within the rDNA (Bryk et al. 1997; Fritze et al. 1997; Smith and Boeke 1997). Like recombination, these functions are intact in other sir mutant cells.

SIR2 is required for silencing at all three major genomic regions in yeast subject to epigenetic control, a role now known to be tied to its catalytic properties as an NAD+-dependent protein deacetylase of histones and other substrates. Significant progress has been made understanding the mechanisms of Sir2p's activities and the broader biological roles for $\operatorname{SIR} 2$ and its relatives in yeast and in organisms ranging from bacteria to vertebrates (for recent reviews, see Blander and Guarente 2004; North and Verdin 2004).

\section{THINKING ABOUT SIR1 MECHANISTICALLY}

Unlike SIR2, SIR3, or SIR4, which act in more than one region of the genome, from early studies, SIRI was distinguished by its apparent restriction to function at the silent mating-type loci. The SIR1 gene can encode a 678 amino acid open reading frame (ORF), with no telltale structural domains or predicted catalytic activities (Stone et al. 1991). However, functional studies define an interaction between Sirlp and the DNA-binding Origin Recognition Complex (ORC; Triolo and Sternglanz 1996; Fox et al. 1997). The discovery of this relationship was particularly appealing because of the longstanding connection between the requirement for passage through $\mathrm{S}$ phase for the reestablishment of silencing after its disruption (Miller and Nasmyth 1984), the role of ORC in silencing (Foss et al. 1993), and the role of Sirlp in establishment. The requirement for both $\mathrm{S}$ phase and ORC for silencing suggested early notions of a requirement for DNA replication in silencing, but these proved incorrect (Kirchmaier and Rine 2001; Li et al. 2001). Models emerging from these observations and others (Chien et al. 1993) suggested that targeting Sir $1 p$ to silenced regions either through tethering to site-specific DNA-binding proteins or through the naturally targeted ORC complex is a critical feature of silent chromatin formation.

Indeed, both a genetic screen and molecular dissection of SIR1 reveal a carboxy-terminal region of Sir1p that is critical for its interaction with Orclp, the largest subunit of ORC, and with Sir4p (Gardner et al. 1999; Bose et al. 2004). These specific interactions, together with the low abundance of Sirlp (Gardner and Fox 2001), may help explain its restricted genomic functions. Furthermore, although Sir1p does not appear to be conserved at the level of primary amino acid sequence in multicellular eukaryotes, the regions identified as critical for interaction with ORC are conserved in other Saccharomyces species, as are two-hybrid interactions between the Saccharomyces bayanus and Saccharomyces mikatae Sir1 proteins and $S$. cerevisiae Orc1p. Tellingly, the most divergent ortholog is from Saccharomyces castellii, and in this case, where five amino acid substitutions are found in the critical interaction region, the two-hybrid interaction is lost (Bose et al. 2004).

Roles beyond transcriptional silencing for Sirlp have been revealed by its occupancy in centromeric chromatin (Sharp et al. 2003). Interestingly, Sir1p appears to act independently of the other Sir proteins, because they are not found in centric chromatin. Furthermore, Sirlp binds to the Caclp chromatin assembly factor and stabilizes its centromeric association. This binding may be functionally significant. Although chromosome stability is normal in single mutants, the sir $1 \Delta \mathrm{cac} 1 \Delta$ double mutant has elevated nondisjunction rates that are further exacerbated when Hirlp, another Cac1p-associated factor, is also mutant.

Unlike larger eukaryotes, Saccharomyces has active genes very close to centromeres. Hence, Sirlp function at the centromeres does not reflect silencing in the sense that $H M L$ and $H M R$ are silenced. However, recombination is suppressed near centromeres, and transcription through centromeres can destroy their function (Panzeri et al. 1984; Snyder et al. 1988). Thus perhaps Sirlp works with other as-yet-undefined proteins to repress recombination at centromeres, possibly in partnership with one of the $H S T$ cousins of SIR2. Alternatively, perhaps it nucleates a nonspreading chromatin structure that helps insulate centromeres from an occasional errant RNA polymerase transit.

\section{ENHANCERS OF $\operatorname{sir} 1 \Delta$ MUTANTS MAKE MORE CHROMATIN CONNECTIONS}

Even in the absence of a detailed understanding of SIRI's molecular mechanism, it seemed likely that the phenotype of the null mutants could give more insight into the epigenetic control of silencing. For example, what protein or process fulfills Sir1p's role in establishment in the fraction of cells that silence $H M L$ and $H M R$ in $\operatorname{sir} 1 \Delta$ mutants?

One approach to answering this question was to identify additional mutants that themselves were mating competent, but in combination with the sir $1 \Delta$ mutation would become completely mating defective (Reifsnyder et al. 1996). In this screen for enhancers of the sirld phenotype, nonnull alleles of SIR2, SIR3, and SIR4 were identified, some of which have been studied in detail and give insight into locus-specific requirements for these genes (Stone et al. 2000; Garcia and Pillus 2002). Significantly, an allele of the $S A S 2$ gene was recovered. Other alleles of $S A S 2$ had been identified independently as suppressors that restore mating to strains bearing a defective, sensitized HMR cis-silencer sequence (Ehrenhofer-Murray et al. 1997). Both studies found that sas2 mutants completely eliminate silencing of telomeric reporter genes. The fact that Sas $2 p$ functions may be positive in the case of sir $1 \Delta$ mutants and at telomeres, but antagonistic at $H M R$, underscores that it has distinct roles depending on the region of the genome where it acts.

Sas $2 p$ is part of the MYST family, so named for its

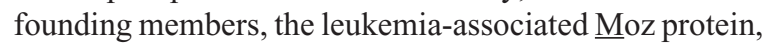

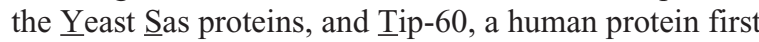
found as an interactor with the HIV Tat transactivator. 
The MYST family is broadly conserved, with two closely related proteins in yeast, Esa1p and Sas3p, and multiple orthologs in every eukaryote for which sequence information is available. Relatively weak sequence similarity to proteins with acetyltransferase activity led to the discovery of histone and nucleosome acetyltransferase activity for all three yeast paralogs and many multicellular orthologs. These orthologs contribute to such diverse processes as dosage compensation, apoptosis, and response to DNA damage (for review, see Carrozza et al. 2003; Utley and Côté 2003).

MYST family proteins, including Sas2p, are found in complexes whose subunits contribute to activity and specificity. In the case of Sas2p's partners, Sas $4 p$ and Sas $5 \mathrm{p}$, the mating defect of $\operatorname{sir} 1 \Delta$ cells is enhanced by mutants of either subunit (Xu et al. 1999a,b).

Other enhancers of $\operatorname{sir} 1 \Delta$ also have connections to chromatin modification. NAT1 and $A R D 1$ are required for mating in sirl mutant backgrounds (Whiteway et al. 1987; Stone et al. 1991). Nat1p and Ard1p are components of an amino-terminal protein acetyltransferase (NatA) with many cellular substrates (for review, see Polevoda and Sherman 2003), including a recently described role acetylating Sir3p and Orc1p (Wang et al. 2004). Further, the sir $1 \Delta$ cacl $1 \Delta$ hir $1 \Delta$ mutant introduced above not only affects centromeres, but is also completely mating defective (Kaufman et al. 1998). The loss of mating in these additional mutant backgrounds underscores the idea that Sirlp activity becomes critical for silencing in many genetically compromised circumstances, including in cells with synthetic versions of silencers (McNally and Rine 1991).

We discovered a circumstance requiring SIR 1 that points to a previously unsuspected role of higher-order structure or topological constraints in silencing. Both of the silent mating-type loci, $H M L$ and $H M R$, and the actively transcribed MAT locus reside on chromosome III. Homologous recombination between $H M L$ and $H M R$ results in circular derivatives of the chromosome (Strathern et al. 1979; Newlon et al. 1991) in which only genetic information between the silent loci and telomeres is lost. Fortunately, there are no essential genes distal to these loci. Thus, strains carrying a large circular chromosome III are fully viable and mating competent, yet have no free chromosome ends or telomeres on this chromosome. We observed that in such strains, mutation of SIR 1 results in loss of mating (Fig. 2). Thus, changing chromosomal structure renders silencing completely dependent on SIR1. The mechanism of this dramatic effect is not yet clear, but may result from topological changes, altered localization, altered replication timing, or other higher-order influences on chromosome structure.

\section{ANALOGS BUT NOT HOMOLOGS OF SIR1}

Given that heterochromatin is common to all eukaryotes, and that SIR2 family members seem to participate in heterochromatin in many if not all of these, it is striking that SIR1 homologs have not been found outside of the Saccharomyces genera. If it is so central to the establish-
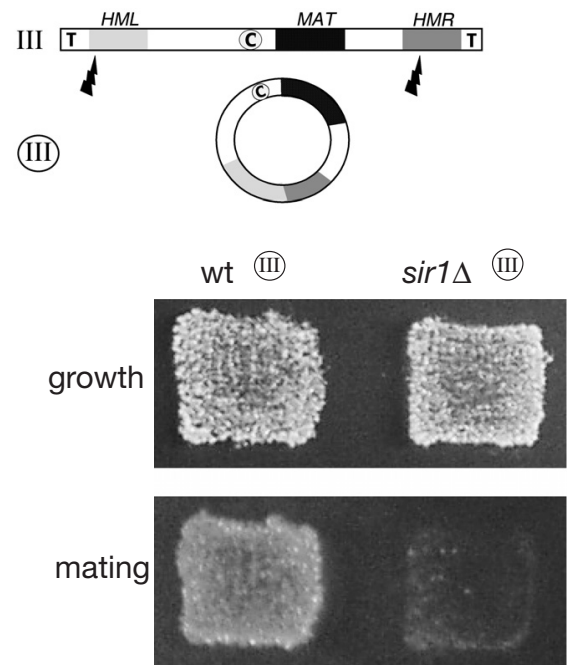

Figure 2. SIR 1 is required for mating-type silencing when chromosome III is circular. Unlike the normal genomic context of a linear chromosome III (as in Fig. 1), sir $1 \Delta$ mutants become completely mating-defective when chromosome III is circularized by recombination between homologous sequences of the silent mating-type loci. Cartoons of chromosome III (top) highlight the telomeres (T), the centromere (C), and the silent mating-type loci $H M L$ and $H M R$, on the left and right arms of the chromosome. Transcription of the centromere-proximal MAT locus defines cell type. In the circular chromosome, recombination has occurred between the sites highlighted by lightning bolts. Although wild-type and sir $1 \Delta$ mutants are healthy with this circular derivative (top), the sir $1 \Delta$ mutants fail to mate (bottom).

ment of heterochromatin in Saccharomyces, what plays this role in other species?

A reasonable argument can be made for HP1 being a Sirlp analog. HP1, so named as the first discovered heterochromatin protein, found initially in Drosophila, is required for heterochromatin function in both flies and in Schizosaccharomyces pombe (for review, see Eissenberg and Elgin 2000; Kellum 2003; Maison and Almouzni 2004). In Drosophila, HP1 binds to the amino terminus of the Orc1 subunit of ORC analogously to the way that Sirlp binds to ORC, and orc mutants in Drosophila function disrupt heterochromatin (Shareef et al. 2001; Prasanth et al. 2004). Moreover, the heterochromatin function of ORC subunits is functionally interchangeable between yeast and Drosophila (Ehrenhofer-Murray et al. 1995; Pak et al. 1997). Hence, there seems to be a deep and conserved connection between proteins that can initiate heterochromatin and ORC. It is curious that a link between $S$. pombe's ORC and heterochromatin has not been made, especially given Sir2p's requirement for heterochromatin formation in S. pombe (Shankaranarayana et al. 2003; Freeman-Cook et al. 2005).

From a different perspective, perhaps it is more surprising that Saccharomyces does use Sir1 and its paralogs to enhance the efficiency of silencing. After all, a mechanism of repression that has no way for being turned on and off would not be useful for regulating genes that need to be turned on under some conditions but not others. This consideration leads us to suggest that Sir protein-based silencing may have evolved without Sir1 to allow for the 
variable yet heritable expression states of certain genes such as those turned on under some conditions but not others, including subtelomeric genes, such as the FLO genes of Saccharomyces and adhesion genes of Candida (B. Cormack, pers. comm.). Sir1 would then be a protein whose function is to push the equilibrium between the assembly of silenced chromatin versus active chromatin decidedly in the direction of silencing for genes whose purpose is not to be expressed, but to serve as a donor in the repair of double-stranded breaks at $M A T$. It will be interesting to see if HP1 serves a similar linchpin role in recruiting intercalary heterochromatin proteins to sites of ORC localization in $S$. pombe and Drosophila.

\section{MORE SIR1s, MORE EPIGENETICS}

The accumulating evidence from sirl $1 \Delta$ mutants and their enhancers demonstrates that loss of SIR 1 sensitizes transcriptional silencing to require other structural and catalytic regulators of chromatin for efficient silencing. Elements of Sirlp's functions at the $H M L$ and $H M R$ silencer elements are clearly related to its recruitment to those sites by Orc1p and Sir4p. Precisely what does Sir1p do when bound? Most current models propose kinetic roles in the assembly of silent chromatin. For example, by providing an extra kilocalorie or so of free energy, Sir1p's interactions with ORC and Sir4p may overcome a kinetic barrier to the formation of silenced chromatin.

Recent studies cited above suggest that Sirlp has a broader spectrum of function than previously suspected. Sirlp's contribution to centromere function is independent of the other Sir proteins (Sharp et al. 2003). Enhancer screens in sir $1 \Delta$ mutants focused on chromosome loss or segregation phenotypes may yield deeper insight into epigenetic aspects of centromere functions in yeast.

It has not yet been established whether the same regions of Sirlp defined for silencer binding are required for its centromeric functions or for functions with the circular derivative of chromosome III. Any differences might help define specificity of targeting and functionally distinct genomic interactions. Indeed, the SIR 1 gene may encode more than one species of protein. Specifically, SIR1 produces two transcripts, the shorter of which is twice as abundant as the longer (Ivy et al. 1986; Stone et al. 1991). The more abundant transcript, if it is translated, would yield an alternative Sir1p isoform with a disrupted Orc1p-Sir4p binding domain (Sparks and Dieckmann 1998). Multiple in-frame methionine codons near the $5^{\prime}$ end of the SIRI ORF amplify the diversity of potential proteins from the locus. Determining whether other Sir1p isoforms have distinct or interfering functions in silencing may provide new insights into previously defined epigenetic states or those that control other less well understood biological processes.

Indeed, epigenetic control of pseudohyphal switching was recently reported to be influenced by the Hstlp and Hst2p members of the Sir2p family (Halme et al. 2004). Genes regulated by these deacetylases are relatively telomere proximal, regions known from several genomewide studies to be particularly sensitive to histone acety- lation state (see, e.g., the review by Millar et al., this volume). It seems reasonable to expect that as understanding of physiological states becomes more fully integrated with knowledge of transcriptional regulation, additional roles will be uncovered for molecules like Sir1p that may have the capacity to buffer epigenetic states. Moreover, the Saccahromyces genus offers unusually favorable opportunities to uncover new roles for Sirlp-like functions as up to four paralogs of SIR1 can be found within its species.

Although the epigenetic aspects of silencing revealed by $\operatorname{sir} 1$ mutations have been the focus of this discussion, the underlying on-or-off nature of silencing is also of interest as it bears on whether repression mechanisms have analog or digital qualities. This issue has received some attention. For example, the GAL1 gene of $S$. cerevisiae is repressed in medium containing glucose by two different repressors, Gal80p and Mig1p. In the absence of Gal80p, glucose repression of GAL1 is graded, whereas in the absence of Mig1p, it is on or off (Biggar and Crabtree 2001). Hence the quality of gene repression in response to a physiological stimulus can be either analog or digital, depending upon which repressor mediates the signal. The studies of SIR1 suggest that mutants with "leaky" phenotypes can be particularly informative regarding mechanisms of repression, but capitalizing on such opportunities requires assays that can be performed robustly at the single-cell level. The versatility of mating-type regulation makes it supremely amenable to such studies.

\section{ACKNOWLEDGMENTS}

We thank J. Game for providing the parental strain with the circular derivative of chromosome III shown in Figure 2. We have greatly enjoyed continuing discussions with our colleagues and their contributions to the development of our ideas and we appreciate comments from $\mathrm{S}$. Jacobson and J. Heilig on this manuscript. It is a particular delight to acknowledge the help of Ira Herskowitz in the early phases of our work on SIRland the continuing impact his legacy makes. Work in our laboratories is occasionally funded by the National Institutes of Health. We appreciate that support.

\section{REFERENCES}

Aparicio O.M., Billington B.L., and Gottschling D.E. 1991. Modifiers of position effect are shared between telomeric and silent mating-type loci in S. cerevisiae. Cell 66: 1279.

Biggar S.R. and Crabtree G.R. 2001. Cell signaling can direct either binary or graded transcriptional responses. EMBO J. 20: 3167.

Blander G. and Guarente L. 2004. The Sir2 family of protein deacetylases. Annu. Rev. Biochem. 73: 417.

Bose M.E., McConnell K.H., Gardner-Aukema K.A., Muller U., Weinreich M., Keck J.L., and Fox C.A. 2004. The origin recognition complex and Sir4 protein recruit Sir1p to yeast silent chromatin through independent interactions requiring a common Sir1p domain. Mol. Cell. Biol. 24: 774.

Bryk M., Banerjee M., Murphy M., Knudsen K.E., Garfinkel D.J., and Curcio M.J. 1997. Transcriptional silencing of Ty1 elements in the RDN1 locus of yeast. Genes Dev. 11: 255.

Carrozza M.J., Utley R.T., Workman J.L., and Côté J. 2003. The 
diverse functions of histone acetyltransferase complexes. Trends Genet. 19: 321.

Chien C.-T., Buck S., Sternglanz R., and Shore D. 1993. Targeting of SIR1 protein establishes transcriptional silencing at $H M$ loci and telomeres in yeast. Cell 75: 531.

Ehrenhofer-Murray A., Rivier D., and Rine J. 1997. The role of Sas2, an acetyltransferase homolog, in silencing and ORC function in Saccharomyces cerevisiae. Genetics 145: 923.

Ehrenhofer-Murray A.E., Gossen M., Pak D.T.S., Botchan M.R., and Rine J. 1995. Separation of origin recognition complex functions by cross-species complementation. Science 270: 1671 .

Eissenberg J.C. and Elgin S.C. 2000. The HP1 protein family: Getting a grip on chromatin. Curr. Opin. Genet. Dev. 10: 204.

Foss M., McNally F.J., Laurenson P., and Rine J. 1993. Origin recognition complex (ORC) in transcriptional silencing and DNA replication in S. cerevisiae. Science 262: 1838.

Fox C.A., Ehrenhofer-Murray A.E., Loo S., and Rine J. 1997. The origin recognition complex, SIR1, and the S phase requirement for silencing. Science 276: 1547.

Freeman-Cook L.L., Gómez E.B., Spedale E.J., Marlett J., Forsburg S., Pillus L., and Laurenson P. 2005. Conserved locusspecific silencing functions of Schizosaccharomyces pombe $\operatorname{sir} 2^{+}$. Genetics 169: (in press).

Fritze C.E., Verschueren K., Strich R., and Esposito R.E. 1997. Direct evidence for SIR 2 modulation of chromatin structure in yeast rDNA. EMBO J. 16: 6495.

Garcia S.N. and Pillus L. 2002. A unique class of conditional sir 2 mutants displays distinct silencing defects in Saccharomyces cerevisiae. Genetics 162: 721 .

Gardner K.A. and Fox C.A. 2001. The Sir1 protein's association with a silenced chromosome domain. Genes Dev. 15: 147.

Gardner K.A., Rine J., and Fox C.A. 1999. A region of the Sir1 protein dedicated to recognition of a silencer and required for interaction with the Orc1 protein in Saccharomyces cerevisiae. Genetics 151: 31.

Gottlieb S. and Esposito R.E. 1989. A new role for a yeast transcriptional silencer gene, SIR2, in regulation of recombination in ribosomal DNA. Cell 56: 771.

Gottschling D.E., Aparicio O.M., Billington B.L., and Zakian V.A. 1990. Position effect at S. cerevisiae telomeres: Reversible repression of Pol II transcription. Cell 63: 751.

Haber J.E. and George J.P. 1979. A mutation that permits the expression of normally silent copies of mating type information in Saccharomyces cerevisiae. Genetics 93: 13.

Halme A., Bumgarner S., Styles C., and Fink G.R. 2004. Genetic and epigenetic regulation of the FLO gene family generates cell-surface variation in yeast. Cell 116: 405 .

Herskowitz I., Strathern J.N., Hicks J.B., and Rine J. 1977. Mating type interconversion in yeast and its relationship to development in higher eukaryotes. ICN-UCLA Symp. Mol. Cell. Biol. (Eukaryotic Genetics Systems) 111: 193.

Ivy J.M., Klar A.J.S., and Hicks J.B. 1986. Cloning and characterization of four SIR genes of Saccharomyces cerevisiae. Mol. Cell. Biol. 6: 688.

Kaufman P.D., Cohen J.L., and Osley M. A. 1998. Hir proteins are required for position-dependent gene silencing in Saccharomyces cerevisiae in the absence of chromatin assembly factor I. Mol. Cell. Biol. 18: 4793.

Kellum R. 2003. HP1 complexes and heterochromatin assembly. Curr. Top. Microbiol. Immunol. 274: 53.

Kirchmaier A.L. and Rine J. 2001. DNA replication-independent silencing in S. cerevisiae. Science 291: 646.

Klar A.J.S., Fogel S., and Macleod K. 1979. MAR1, a regulator of HMa and HM $\alpha$ loci in Saccharomyces cerevisiae. Genetics 93: 37.

Li Y.C., Cheng T.H., and Gartenberg M.R. 2001. Establishment of transcriptional silencing in the absence of DNA replication. Science 291: 650 .

Mahoney D., Marquardt R., Shei G., Rose A., and Broach J. 1991. Mutations in the HML E silencer of Saccharomyces cerevisiae yield metastable inheritance of trancriptional repression. Genes Dev. 5: 605.

Maison C. and Almouzni G. 2004. HP1 and the dynamics of het- erochromatin maintenance. Nat. Rev. Mol. Cell Biol. 5: 296.

McNally F.J. and Rine J. 1991. A synthetic silencer mediates SIR-dependent functions in Saccharomyces cerevisiae. Mol. Cell. Biol. 11: 5648.

Miller A.M. and Nasmyth K.A. 1984. Role of DNA replication in the repression of silent mating type loci in yeast. Nature 312: 247.

Newlon C.S., Lipschitz L.R., Collins I., Deshpande A., Devenish R.J., Green R.P., Klein H.L., Palzkill T.G., Ren R., Synn S., and Woody S.T. 1991. Analysis of a circular derivative of Saccharomyces cerevisiae chromosome III: A physical map and identification and location of ARS elements. Genetics 129: 343.

North B.J. and Verdin E. 2004. Sirtuins: Sir2-related NAD-dependent protein deacetylases. Genome Biol. 5: 224

Pak D.T.S., Pflumm M., Chesnokov I., Huang D.W., Kellum R., Marr J., Romanowski P., and Botchan M.R. 1997. Association of the origin recognition complex with heterochromatin and HP1 in higher eukaryotes. Cell 91: 311.

Panzeri L., Groth-Clausen I., Shepard J., Stotz A., and Phillippsen P. 1984. Centromeric DNA in yeast. Chromosomes Today 8: 46.

Pillus L. and Rine J. 1989. Epigenetic inheritance of transcriptional states in S. cerevisiae. Cell 59: 637.

Polevoda B. and Sherman F. 2003. Composition and function of the eukaryotic N-terminal acetyltransferase subunits. Biochem. Biophys. Res. Commun. 308: 1.

Prasanth S.G., Prasanth K.V., Siddiqui K., Spector D.L., and Stillman B. 2004. Human Orc2 localizes to centrosomes, centromeres and heterochromatin during chromosome inheritance. $E M B O$ J. 23: 2651.

Reifsnyder C., Lowell J., Clarke A., and Pillus L. 1996. Yeast $S A S$ silencing genes and human genes associated with AML and HIV-1 Tat interactions are homologous with acetyltransferases. Nat. Genet. 14: 42.

Rine J. and Herskowitz I. 1987. Four genes responsible for a position effect on expression from HML and HMR in Saccharomyces cerevisiae. Genetics 116: 9.

Rine J., Strathern J.N., Hicks J.B., and Herskowitz I. 1979. A suppressor of mating-type locus mutations in Saccharomyces cerevisiae: Evidence for and identification of cryptric matingtype loci. Genetics 93: 877.

Rusche L.N., Kirchmaier A.L., and Rine J. 2003. The establishment, inheritance, and function of silenced chromatin in Saccharomyces cerevisiae. Annu. Rev. Biochem. 72: 481.

Shankaranarayana G.D., Motamedi M.R., Moazed D., and Grewal S.I. 2003. Sir2 regulates histone H3 lysine 9 methylation and heterochromatin assembly in fission yeast. Curr. Biol. 13: 1240.

Shareef M.M., King C., Damaj M., Badagu R., Huang D.W., and Kellum R. 2001. Drosophila heterochromatin protein 1 (HP1)/origin recognition complex (ORC) protein is associated with HP1 and ORC and functions in heterochromatin-induced silencing. Mol. Biol. Cell 12: 1671.

Sharp J.A., Krawitz D.C., Gardner K.A., Fox C.A., and Kaufman P.D. 2003. The budding yeast silencing protein Sir1 is a functional component of centromeric chromatin. Genes Dev. 17: 2356 .

Smith J.S. and Boeke J.D. 1997. An unusual form of transcriptional silencing in yeast ribosomal DNA. Genes Dev. 11: 241.

Snyder M., Sapolsky R.J., and Davis R.W. 1988. Transcription interferes with elements important for chromosome maintenance in Saccharomyces cerevisiae. Mol. Cell. Biol. 8: 2184.

Sparks K.A. and Dieckmann C.L. 1998. Regulation of poly(A) site choice of several yeast mRNAs. Nucleic Acids Res. 26: 4676.

Stone E.M., Reifsnyder C., McVey M., Gazo B., and Pillus L. 2000. Two classes of sir 3 mutants enhance the sirl mutant mating defect and abolish telomeric silencing in Saccharomyces cerevisiae. Genetics 155: 509.

Stone E.M., Swanson M.J., Romeo A.M., Hicks J.B., and Sternglanz R. 1991. The SIR1 gene of Saccharomyces cerevisiae and its role as an extragenic suppressor of several mating-defective mutants. Mol. Cell. Biol. 11: 2253. 
Strathern J.N., Newlon C.S., Herskowitz I., and Hicks J.B. 1979. Isolation of a circular derivative of yeast chromosome III: Implication for the mechanism of mating type interconversion. Cell 18: 309.

Triolo T. and Sternglanz R. 1996. Role of interactions between the origin recognition complex and SIR1 in transcriptional silencing. Nature 381: 251.

Utley R.T. and Côté J. 2003. The MYST family of histone acetyltransferases. Curr. Top. Microbiol. Immunol. 274: 203.

Wang X., Connelly J.J., Wang C.L., and Sternglanz R. 2004. Importance of the Sir3 $\mathrm{N}$ terminus and its acetylation for yeast transcriptional silencing. Genetics 168: 547.

Whiteway M., Freedman R., Arsdell S.V., Szostak J.W., and Thorner J. 1987. The yeast $A R D 1$ gene product is required for repression of cryptic mating-type information at the $H M L$ locus. Mol. Cell. Biol. 7: 3713.

Xu E.Y., Kim S., and Rivier D.H. 1999a. SAS4 and SAS5 are locus-specific regulators of silencing in Saccharomyces cerevisiae. Genetics 153: 25.

Xu E.Y., Kim S., Replogle K., Rine J., and Rivier D.H. 1999b. Identification of SAS4 and SAS5, two genes that regulate silencing in Saccharomyces cerevisiae. Genetics 153: 13. 


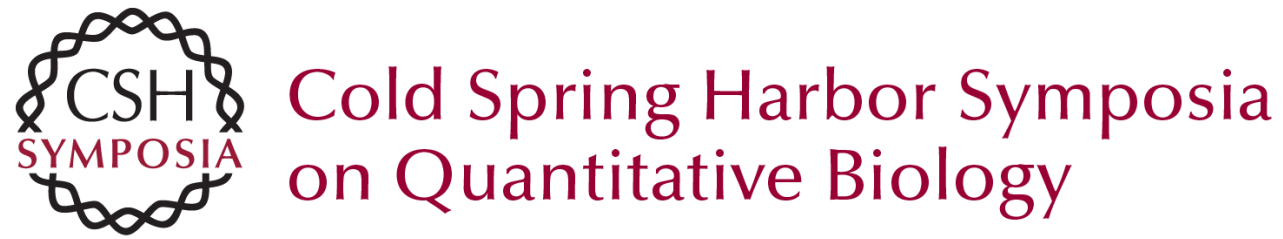

\section{SIR1 and the Origin of Epigenetic States in Saccharomyces cerevisiae}

L. PILLUS and J. RINE

Cold Spring Harb Symp Quant Biol 2004 69: 259-266

Access the most recent version at doi:10.1101/sqb.2004.69.259

References This article cites 57 articles, 33 of which can be accessed free at: http://symposium.cshlp.org/content/69/259.full.html\#ref-list-1

License

Email Alerting Receive free email alerts when new articles cite this article - sign up in Service the box at the top right corner of the article or click here. 